Digital engagement metrics: 863,931 impressions, 1\% Cut Through Rate for linked advertisements, $0.05 \%$ social engagement, and 5,000 peer educator conversations with campaign recall increase of $25 \%$. Pre \& post-intervention surveys showed increased social norms for STI testing (24\% increase), positive attitudes (26\%), and intention to STI test in the next 12 months (32\%). Over $80 \%$ of participants were within the two high-risk groups.

Conclusion Down to Test successfully engaged high risk young people attending music festivals and improved healthy sexual behaviour facilitators. Applying segmentation research and engaging the identified groups improved the likelihood of success by better targeting the activations. Broad reach across NSW was maximised by using festivals in a variety of locations. The program is adaptable to other locations.

Disclosure No significant relationships.

\section{P352 STD, HIV, AND PREGNANCY TESTING BEHAVIORS AMONG INTERNET AND MOBILE DATING APPLICATION USERS AND NON-USERS, 2016}

${ }^{1}$ Alexandra Coor, ${ }^{2}$ Matthew Hogben. ${ }^{1}$ Centers for Disease Control and Prevention, Division of STD Prevention, Atlanta, USA; ${ }^{2}$ Centers for Disease Control and Prevention, Atlanta, USA

10.1136/sextrans-2019-sti.459

Background Use of internet websites and geosocial networking mobile applications for sexual and romantic relationships has grown steadily. We examined the prevalence of dating app use and STD, HIV and pregnancy testing among market research survey respondents.

Methods We analyzed 2015-2016 data from the Scarborough/ MARS Healthcare Module, which contains data from two market research data sets. In this sample, 199,308 responses were received ( $18 \geq$ years), weighted, combined across datasets, and projected to a 2016 U.S. adult population. We also assessed dating app use by demographics, STD, HIV, and pregnancy testing practices in the past year.

Results Of the projected population ( $\mathrm{n}=197,150,967), 6.2 \%$ (7.0\% men; $5.3 \%$ women) reported using dating apps in the past 30 days. Over half of all dating app users (50.3\%) were between the ages of 18-34 years. App users were more likely than non-users to report male gender (55.2\% vs $48.1 \%)$, Hispanic ethnicity $(29.4 \% \mathrm{v} 16.2 \%)$ and race other than white $(31.3 \%$ v 23.1\%). Fifty-seven percent of dating app users were never married, and $23.8 \%$ were married at the time of use. Overall, a higher proportion of dating app users than non-users reported STD testing $(6.5 \%$ vs $4.0 \%, P R=1.64)$ and HIV testing $(5.9 \%$ vs $3.8 \%, \mathrm{PR}=1.54)$, in the past year. However, among 18-24 year olds, STD and HIV testing was 14$16 \%$ less common among users, compared to non-users. Women using dating apps were more likely than non-users to take pregnancy tests in the past year $(8.8 \%$ vs $5.6 \%$, $\mathrm{PR}=1.57$ ).

Conclusion A small proportion of the general population use dating apps, and STD testing rates were low. However, efforts to build upon the higher rates of reported testing for STD, HIV, and pregnancy among app users (e.g., site advertisements, questions during sexual healthcare visits) might improve testing rates in this group. Targeted attention to adolescents and young adults is warranted.

Disclosure No significant relationships.
P353 SOCIAL APPS AND THE EVOLVING RISK ENVIRONMENT: A CROSS-SECTIONAL SURVEY AMONG MEN WHO ATTEND STDS CLINICS IN GUANGDONG, CHINA

${ }^{1}$ Changchang Li, 'Lei Chen, 'Weiming Tang, 'Bin Yang, ${ }^{2}$ He-Ping Zheng, ${ }^{1}$ Cheng Wang. ${ }^{1}$ Dermatology Hospital of Southern Medical University, Guangzhou, China; ${ }^{2}$ Dermatology Hospital of Southern Medical University, Guangdong Center for STD Control and Prevention, Guangzhou, China

\subsection{6/sextrans-2019-sti.460}

Background Social apps provided important channels for people to communicate with each other. However, social network apps use also created a unique chance for casual partner seeking. This study aimed to know the situation of the using of social apps for partner seeking purpose among men who attend STDsclinics (MSCs) and to describe sexual behaviors among users.

Methods We conducted a cross-sectional study amongMSCs recruited from 9 cities in Guangdong, China from March to August in 2018. Data on socio-demographics, sexual behaviors and social app use for sex-seekingwere collected. Multivariable logistic regression models were used to identify the factors associated withpartner seeking through social apps.

Results A total of 1954 participants were recruited, with a mean age of 39.9 (SD: 13.9). Overall, 228 (11.7\%) ever used a mobile app for partner-seeking. Among people seeking partner through social apps, $36.6 \%$ have one more partners, $14.0 \%$ met their partnersin-person within 24 hours, and $33.8 \%$ engaged incondomless intercourse with the last partner found through social apps. Only 27.2\% of them negotiated about condom use before meeting in-person, and $12.3 \%$ asked for HIV status of the last partner before meeting in-person. Sex-seeking appsuse was positively associated with having casual partners in the last three months $(\mathrm{aOR}=3.5$, 95\% CI 2.64.7), everhaving anal sex with men $(\mathrm{aOR}=21.5$, 95\%CI $10.6-$ 43.6), and not receiving HIV prevention services $(\mathrm{aOR}=7.9$, 95\%CI 5.6-11.3). Condomless intercourse with the last partner was positively associated with having more than three partners from app $(\mathrm{aOR}=5.0,95 \% \mathrm{CI} 1.9-13.2)$ and negotiating about condom use with the partner before meeting in-person $(\mathrm{aOR}=2.2$, 95\% CI 1.2-4.4).

Conclusion Social apps use has become an important sex-seekingroute among Chinese MSCs, which may facilitate the transmission of STDs. Social apps-based interventions are urgently needed.

Disclosure No significant relationships.

\section{P356 HIV-RELATED STIGMA \&AMP; DISCRIMINATION IN WESTERN NIGERIA: EXPERIENCES OF PEOPLE LIVING WITH HIV \&AMP; RIGHTS ISSUES}

${ }^{1}$ Saheed Usman, ${ }^{2}$ Ibiwumi Usman*. 'APIN Public Health Intiatives, Abuja, Nigeria; ${ }^{2}$ Kids and Teens Resource Centre, Akure, Nigeria

\subsection{6/sextrans-2019-sti.461}

Background HIV-related stigma and discrimination continue to be major social determinants driving the epidemic of HIV globally despite the advances in medical treatment and increases in the awareness. Hypotheses tested was right awareness of people living with HIV/AIDS influencing HIV-related stigma \& discrimination. The study aimed at assessing the level of HIV/AIDS related stigma and discrimination, forms, 
effects, and internal stigma experienced by PLHIVs in SouthWestern Nigeria.

Methods This cross sectional study was carried out at eight PEPFAR supported primary, secondary and tertiary level hospitals in South-Western Nigeria. The target population was adult (18 years and above) male and female persons living with HIV (PLHIVs) including key population. Data was collected from 278 consenting respondents by trained volunteers by a face-to-face interview.

Results The mean age \pm SD of the respondents was $38.48 \pm$ 11.48 years, $70.05 \%$ females, mostly married in a monogamous setting (48.6\%), with a formal education $(86.3 \%)$, traders $(33.5 \%)$, live in rural area $(88.5 \%)$ while people in the key populations accounted for $9.4 \%$ of the participants. 78.4\% elicited negative feelings such as depression and shame after diagnosis. About one-third (33.1\%) PLHIVs have ever experienced HIV-related stigma and discrimination mostly gossip, physical abuse, and verbal insult, of which about twothird $(63.2 \%)$ occurred in the hospital setting, followed by home/community (25.0\%). In addition, $8.6 \%$ have been refused a job while $5.0 \%$ have lost their job because of their HIV status. Rights awareness by PLHIVs does not rule out HIV-related stigma \& discrimination experience $\left(\chi^{2}=5.29\right.$, $\mathrm{df}=1, \mathrm{P}=0.021$ ).

Conclusion A remarkable proportion of PLHIV still face stigma/discrimination with possible dramatic impact on their treatment and resultant quality of life. Efforts therefore, should be made to ensure PLHIV are not only aware of their rights, but are empowered to seek redress if these rights are violated.

Disclosure No significant relationships.

\section{P357 INTERLINKING STIGMA, VIOLENCE AND STI/HIV AMONG INJECTING DRUG USERS IN INDIA}

Santosh Sharma*. International Institute for Population Sciences, Population Studies, Mumbai, India

\subsection{6/sextrans-2019-sti.462}

Background Injecting drug users (IDUs) are at higher risk of acquiring sexually transmitted infections (STIs) and untreated STIs makes IDUs and their partners vulnerable to HIV infection in India especially in case of existing stigma/discrimination and violence against them. This study aims to interlink the stigma, violence and STI/HIV among IDUs.

Methods Data from Integrated biological and behavioural survey (IBBS), 2014-15, conducted among male IDUs in India, has been used in this study. IDUs in IBBS were operationally defined as Men, aged 15 years or more, who has used any psychotropic (addictive/mind altering) substance or drug for recreational or non-medical reasons through injections, at least once in the last 3 months. A total of 3175 interviewed IDUs from Manipur, Nagaland and Maharashtra has been analysed by descriptive and multivariate analysis.

Results Portray that Widowed/divorced/separated IDUs, those who were living alone, and those who had initiated injecting drug use below age 18 years were more likely to suffer general stigma, stigma at health facility and physical violence. IDUs those who have started injecting drug within the first year of drug use were more likely to suffered stigma at health facility (35\%) and physical violence (32\%). Prevalence of STI was higher among those IDUs who were facing the general stigma (19.2\%), stigma at health facility (21.4\%) and physically beaten $(26 \%)$ because of their IDUs status. Multivariate analysis revealed that IDUs who were facing general stigma, violence and experienced at least one STI symptom were significantly $1.33 \quad(\mathrm{p}<0.05)$ times, $1.98 \quad(\mathrm{p}<0.001)$ and 1.06 $(\mathrm{p}<0.05)$ times more likely to have HIV.

Conclusion Existing HIV prevention programmes and service providers should have addressed the existing stigma/discriminations and violence associated with their STI/HIV status among IDUs in India. The critical groups of IDUs needs urgent programme to address the micro and macro level determinants that shape their risk environments.

Disclosure No significant relationships.

\section{P360 IMPROVING HEALTH CARE WORKER UNDERSTANDING OF LGBTQ+ PATIENTS THROUGH STORYTELLING AND EMPATHY}

${ }^{1}$ Amanda Long, ${ }^{1}$ Errol Fields*, ${ }^{1}$ Aruna Chandran, ${ }^{2}$ Kehinde Bademosi, ${ }^{1}$ Simone Sawyer, ${ }^{1}$ Jeannie Murray, ${ }^{3}$ Christina Schumacher, ${ }^{2}$ Adena Greenbaum, ${ }^{1}$ Jacky Jennings. 'Johns Hopkins University School of Medicine, Center for Child and Community Health Research (CCHR), Baltimore, USA; ${ }^{2}$ Baltimore City Health Department, Baltimore, USA; ${ }^{3}$ Johns Hopkins School of Medicine, Baltimore, USA

\subsection{6/sextrans-2019-sti.463}

Background Significant HIV disparities affect LGBTQ+ populations and medical mistrust from experiences of healthcare worker (HCW) stigma is a key barrier to HIV prevention and care. This stigma results from many factors, including lack of HCW understanding of LGBTQ+ experiences. The goal was to determine if storytelling nights, events where LGBTQ+ persons share in-depth, personal accounts of their experiences, increased HCW understanding of LGBTQ+ patients.

Methods The study design was a retrospective post-test design with surveys conducted after each of 5 storytelling nights and qualitative data collected via post-it notes during each event. All city residents were encouraged to attend, with marketing focused on the LGBTQ+ community and HCWs that interact with LGBTQ+ patients. The survey measured changes in HCW understanding of LGBTQ+ patients as a result of the event. Post-it notes measured HCW reactions to LGBTQ+ patient experiences shared through the storytelling and were analyzed using thematic analysis.

Results 416 individuals attended the storytelling nights (mean 83, SD 12.7 per event) and 56 HCWs completed a survey. $49(87.5 \%)$ strongly agreed or agreed that they could better understand their patients' feelings and concerns after the event. 449 post-its (mean 90, SD 23.1 per event) were collected and $43(9.6 \%)$ post-its were directly related to HCW reflections on LGBTQ+ patients. Three themes emerged from post-it responses: learning new approaches for working with LGBTQ+ patients through the stories; storytelling nights meeting an educational need for HCWs working with LGBTQ+ patients; and the realization that HCWs should take more time to learn about each patient's needs.

Conclusion Events that encourage sharing of experiences, such as storytelling nights, may serve as an effective tool for increasing understanding by HCWs of LGBTQ+ patients by the sharing of unique experiences of LGBTQ+ persons. This may ultimately help to decrease medical distrust of LGBTQ+ populations and improve sexual health and well-being.

Disclosure No significant relationships. 